\title{
A new method of preparing single-walled carbon nanotubes ${ }^{\text {II }}$
}

\author{
S R C VIVEKCHAND ${ }^{1}$ and A GOVINDARAJ ${ }^{1,2, *}$ \\ ${ }^{1}$ Chemistry and Physics of Materials Unit, Jawaharlal Nehru Centre for \\ Advanced Scientific Research, Jakkur PO, Bangalore 560 064, India \\ ${ }^{2}$ Solid State and Structural Chemistry Unit, Indian Institute of Science, \\ Bangalore 560 012, India \\ e-mail: govind@jncasr.ac.in
}

\begin{abstract}
A novel method of purification for single-walled carbon nanotubes, prepared by an arc-discharge method, is described. The method involves a combination of acid washing followed by high temperature hydrogen treatment to remove the metal nanoparticles and amorphous carbon present in the as-synthesized singlewalled carbon nanotubes. The purified single-walled carbon nanotubes have been characterised by low-angle X-ray diffraction, electron microscopy, thermo-gravimetric analysis and Raman spectroscopy.
\end{abstract}

Keywords. Nanotubes; synthesis; purification.

\section{Introduction}

Carbon nanotubes have interesting properties due to their one-dimensional structure and giant molecular nature. ${ }^{1,2}$ A single-walled carbon nanotube (SWNT) can be either metallic or semi-conducting depending on the diameter and chirality. ${ }^{3}$ Single-walled carbon nanotubes were discovered ${ }^{4,5}$ in 1993 and large quantities of SWNTs have been prepared by both laser ablation and arc discharge methods. ${ }^{6,7}$ SWNTs have also been prepared by the pyrolysis of hydrocarbons on nanometer sized metal catalysts. ${ }^{1,2,8}$ The SWNTs prepared by these procedures contain impurities like fullerenes, metal catalyst particles that are most often coated with layers of carbon, generally in the amorphous form. The raw SWNTs samples have been purified by various methods. ${ }^{9-18}$ Hydrothermal treatment has been used by Tohji et $a l^{9,10}$ to remove the amorphous carbon from the nanotube surface. Smalley and coworkers ${ }^{1-13}$ have used micro-filtration to eliminate the amorphous carbon and catalyst particles. Fullerenes have been removed by solvent extraction. Most of the procedures employ acid treatment to dissolve the metal particles and air oxidation for removing the amorphous carbon. ${ }^{9-18}$ Margrave and coworkers ${ }^{16,17}$ have employed gas-phase purification to obtain pure SWNTs. Martinez et al ${ }^{18}$ have used microwave acid digestion to reduce the time taken for the purification. High temperature annealing of the purified samples has been adopted by some procedures. ${ }^{12,15}$ This removes the chemical functional groups created on the nanotube surface due to acid treatment. $^{1,2}$ It is also important to note that the purification procedure employed is sample dependent. ${ }^{15}$ There are only a few procedures to purify SWNTs prepared by the arc discharge method. ${ }^{9,10,18}$ In this paper we report a simple and novel procedure to purify

\footnotetext{
IIDedicated to Professor C N R Rao on his 70th birthday

*For correspondence
} 
SWNTs produced by the arc discharge method. ${ }^{7}$ We have combined air oxidation, acid treatment and high temperature hydrogen etching to obtain highly pure SWNT samples. The high temperature hydrogen treatment helps in the removal of amorphous carbon simultaneously the SWNTs are also annealed. We have used low-angle powder X-ray diffraction, electron microscopy, thermogravimetric analysis and Raman spectroscopy to characterize the sample purity.

\section{Experimental}

The SWNTs for the experiments were prepared by the method as reported by Journet et $a l{ }^{7}$ Briefly, the SWNTs were synthesized by the DC arc discharge method using a composite rod containing $\mathrm{Y}_{2} \mathrm{O}_{3}(1$ at.\%) and $\mathrm{Ni}(4.2$ at.\%) as the anode and a graphite rod as the cathode, under a helium pressure of 660 torr with a current of $100 \mathrm{~A}$ and $30 \mathrm{~V}$. The nanotubes were heated in air at $573 \mathrm{~K}$ for $12 \mathrm{~h}$ to remove the amorphous carbon coating on the catalyst particles. The SWNT sample was then stirred in conc. $\mathrm{HNO}_{3}$ at $333 \mathrm{~K}$ for $24 \mathrm{~h}$ in order to dissolve the metal nanoparticles. The sample was washed with distilled water, dried and dispersed in ethanol under sonication and filtered using Millipore $(0 \cdot 3 \mu \mathrm{m})$ filter paper, the SWNTs were collected on the filter paper. Later, this was dried in the oven at $373 \mathrm{~K}$ for $2 \mathrm{~h}$. The sample was heated in a furnace at $1473 \mathrm{~K}$, in flowing hydrogen at $100 \mathrm{sccm}$ (standard cubic centimetre per minute) for $2 \mathrm{~h}$. This SWNTs sample was again stirred in conc. $\mathrm{HNO}_{3}$ at $333 \mathrm{~K}$ for $3 \mathrm{~h}$ and finally heated in a furnace at $1573 \mathrm{~K}$ for $2 \mathrm{~h}$ in flowing hydrogen $(100 \mathrm{sccm})$. The overall scheme of purification is illustrated in figure 1.

Functionalized SWNTs were obtained in three steps. In the first step, acid treated SWNTs (sample B) were refluxed in a mixture of conc. $\mathrm{HCl}$ and $\mathrm{H}_{2} \mathrm{O}_{2}$ for $30 \mathrm{~min}$. In the

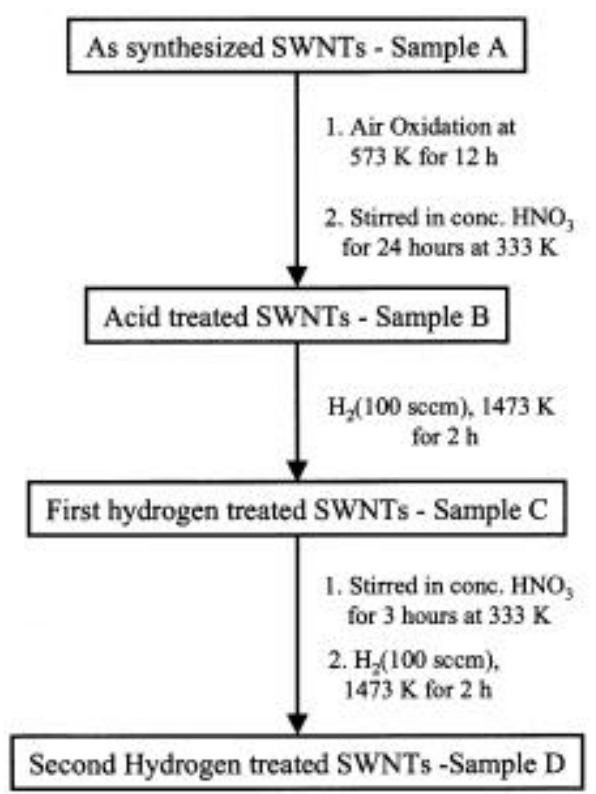

Figure 1. Schematic representation of the purification procedure. 
second step the acid-fuctionalized SWNTs are refluxed in $\mathrm{SOCl}_{2}$ in an argon atmosphere overnight to obtain chlorinated SWNTs. In the third step, butylamine/hexylamine was stirred with chlorinated SWNTs for $72 \mathrm{~h}$ to obtain amine functionalized SWNTs.

The nanotubes were characterized at each stage by powder X-ray diffraction, Scanning Electron Microscopy (SEM), Transmission Electron Microscopy (TEM) and Raman spectroscopy. The powder X-ray diffraction patterns was recorded using a Seifert XRD 3000 TT instrument. All the SEM images were obtained with a Leica S-440I microscope and TEM images with a JEOL JEM 3010 instrument operating at an accelerating voltage of $300 \mathrm{kV}$. The Raman measurements were performed in a $90^{\circ}$ geometry using a Jobin Yvon TRIAX 550 triple grating spectrometer equipped with a cryogenic charge-coupled device camera, using diode-pumped frequency doubled solid state Nd:YAG laser of $532 \mathrm{~nm}$ (Model DPSS 532-400, Coherent Inc. USA).

\section{Results and discussions}

In figure 2a, we show the SEM image of the as-synthesized SWNTs (sample A). Sample A contains amorphous carbon, particles and SWNT bundles. The TEM image in figure

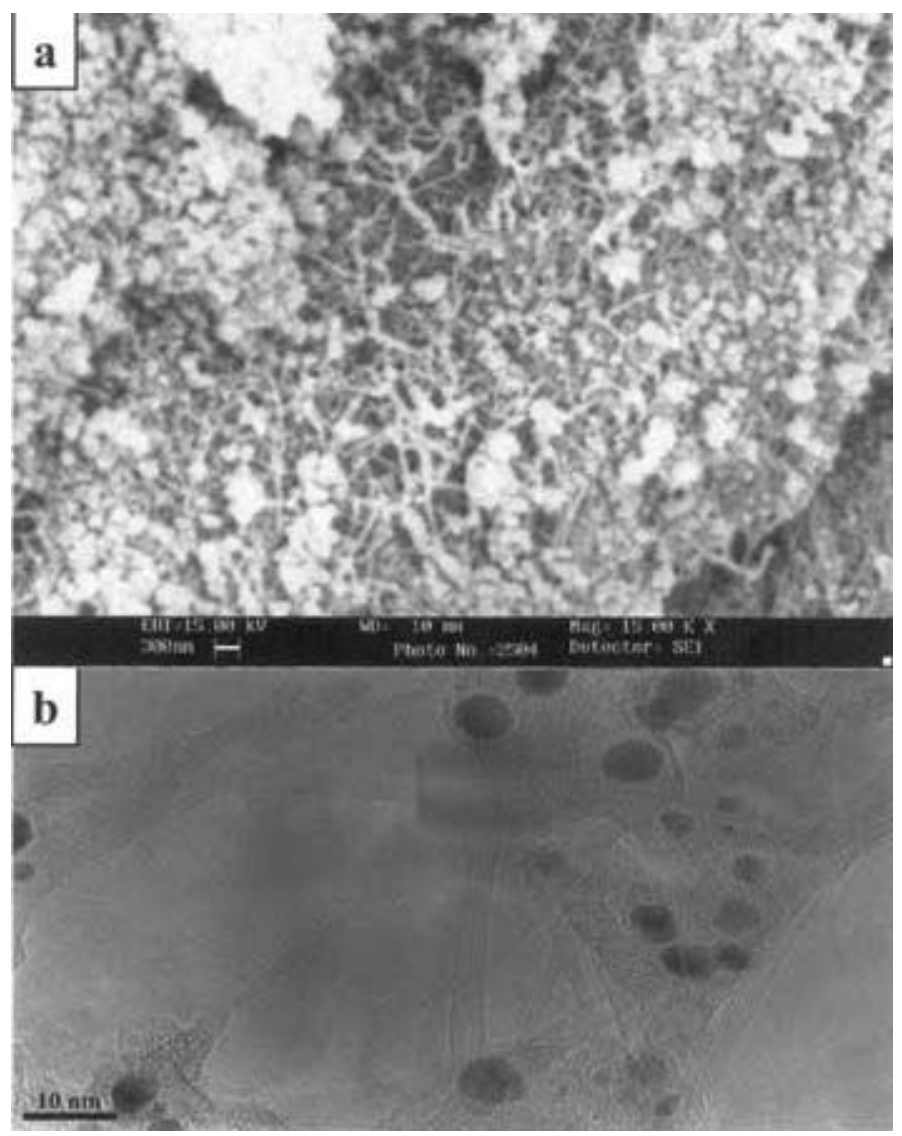

Figure 2. (a) SEM image and (b) TEM image of as-synthesised SWNTs (sample A). 

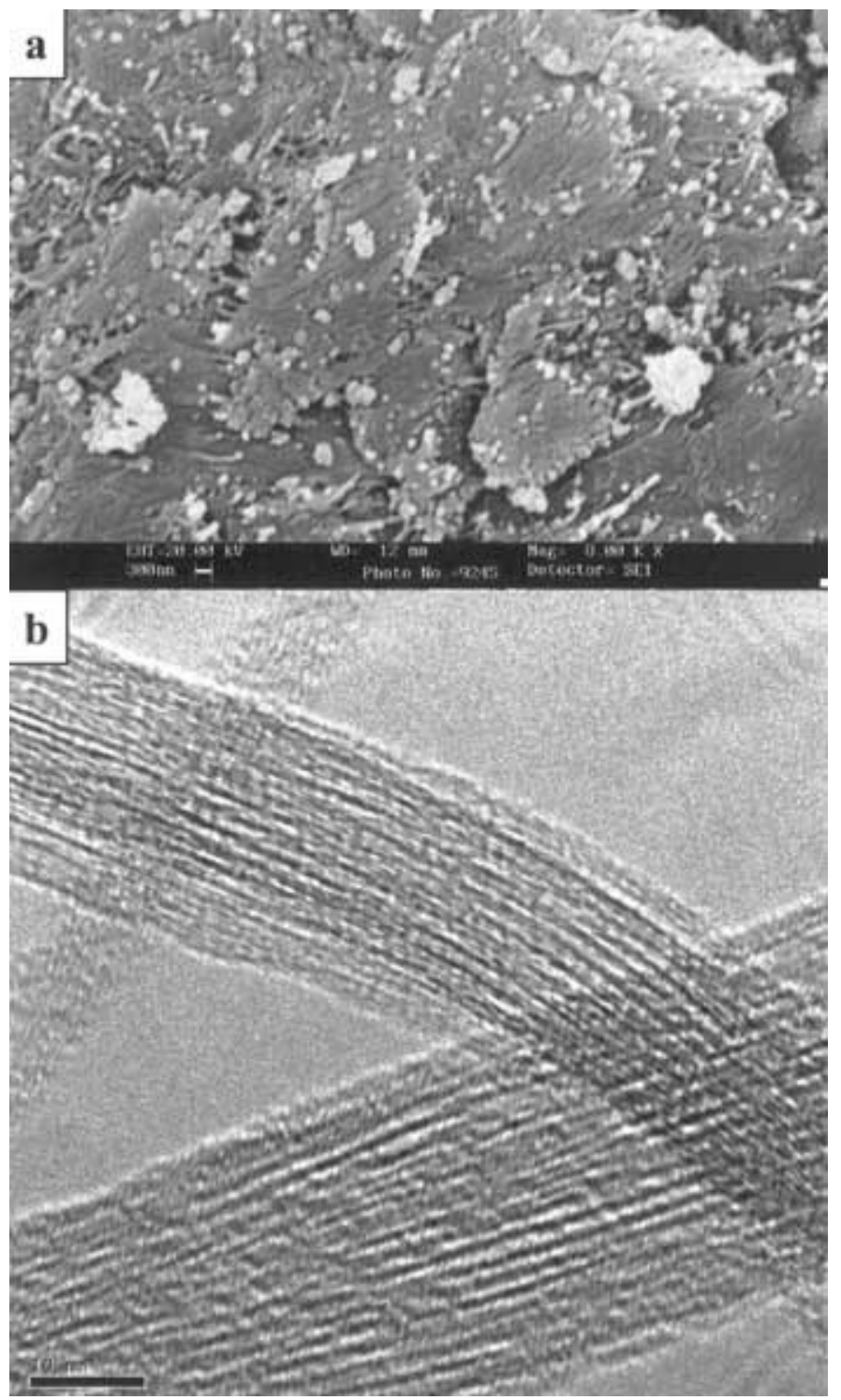

Figure 3. (a) SEM image and (b) TEM image of acid-treated SWNTs (sample B).

$2 \mathrm{~b}$ shows that the particles are metal catalyst covered with carbon. The carbon coating on the metal particles prevents the dissolution of the metal in acid. We employed air oxidation of SWNT samples at $573 \mathrm{~K}$ for $12 \mathrm{~h}$ to remove the carbon coating on the metal nanoparticles. Oxidation of SWNTs is catalysed by the metal nanoparticles and occurs above $600 \mathrm{~K}^{17}$ The subsequent acid treatment removes the metal particles as seen from figure 3 (sample B). Both the SEM and TEM images show that there is covering of amorphous carbon on the SWNTs. 


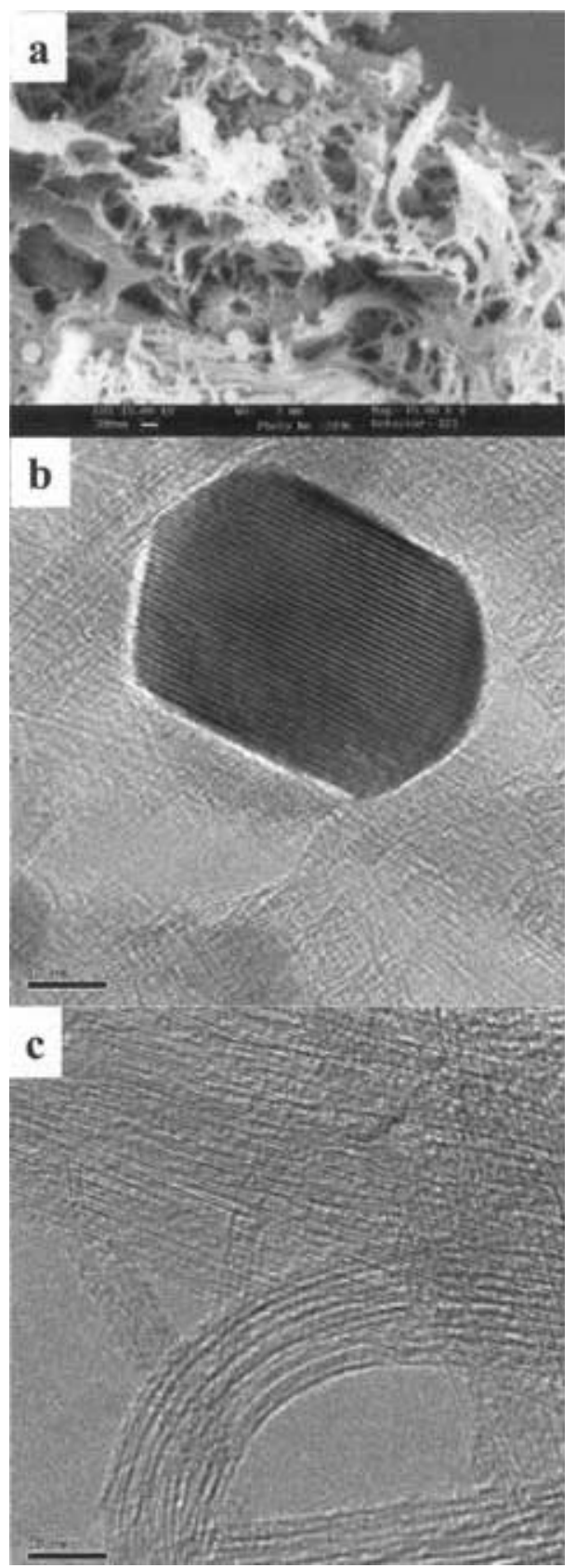

Figure 4. (a) SEM image, (b) and (c) TEM images of SWNTs samples after first hydrogen treatment (sample $\mathrm{C}$ ).

The first hydrogen-treated samples (sample C) contain primarily SWNTs and a few metal particles as seen in figures $4 \mathrm{a}$ and $\mathrm{b}$. The SEM image of the hydrogen-treated sample in figure $4 \mathrm{a}$ shows SWNT bundles with diameters $20-50 \mathrm{~nm}$. The metal particles 
are large and single crystalline as seen in figure $4 \mathrm{~b}$. Figure $4 \mathrm{c}$ shows the presence of high quality SWNTs obtained after hydrogen treatment. From the TEM images we have calculated the SWNT diameter distribution to be in the $1.3-1.9 \mathrm{~nm}$ range. It is well known that the melting points of small clusters are much lower than the bulk. In bulk, nickel (m.p. 1726 K) and yttrium (m.p. $1795 \mathrm{~K}$ ) melt above the temperatures employed

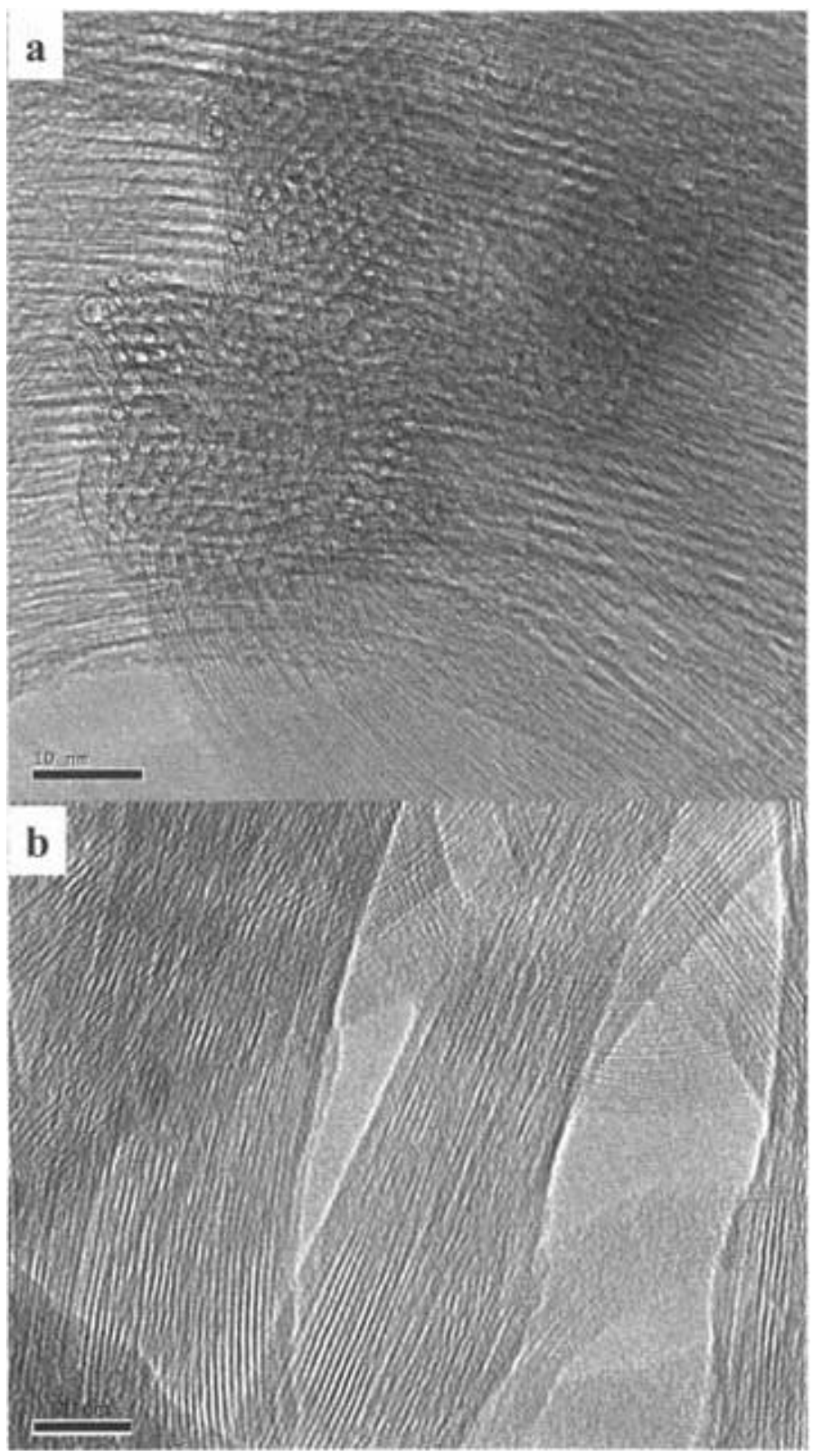

Figure 5. (a) and (b) TEM images of purified SWNTs (sample D). 
in our purification procedure. In this case the melting and agglomeration of the small metal nanoparticles leads to the formation of large metal particles. The presence of large metal particles indicates that the step 1 in the purification procedure is not $100 \%$ efficient. We have found that these metal particles have no amorphous or graphitic carbon covering the surface.

The metal particles are removed by repeating the acid treatment for a shorter duration and another high temperature hydrogen treatment to yield pure SWNTs (sample D). The TEM images of sample D is shown in figure 5. In figure 5a, one can see the cross the cross-sectional view of the SWNTs. The TGA analysis of this SWNT sample shows the sample contains negligible impurities after second hydrogen treatment.

In other procedures employed for SWNTs (arc-discharge) purification, the final purified SWNTs contain hollow onion-like structures. ${ }^{9,10,18}$ The hollow onion-like structures are obtained when the metal in the carbon-coated metal nanoparticles gets dissolved in conc. $\mathrm{HNO}_{3}$ during the purification. We did not observe such onion structures in the purified SWNTs prepared by us, indicating that carbon covering on the metal particles is etched away by hydrogen.

SWNTs form a triangular lattice, which gives a characteristic low angle reflection in the XRD pattern. ${ }^{6}$ In figure 6 , the low-angle X-ray diffraction patterns of as-synthesized,

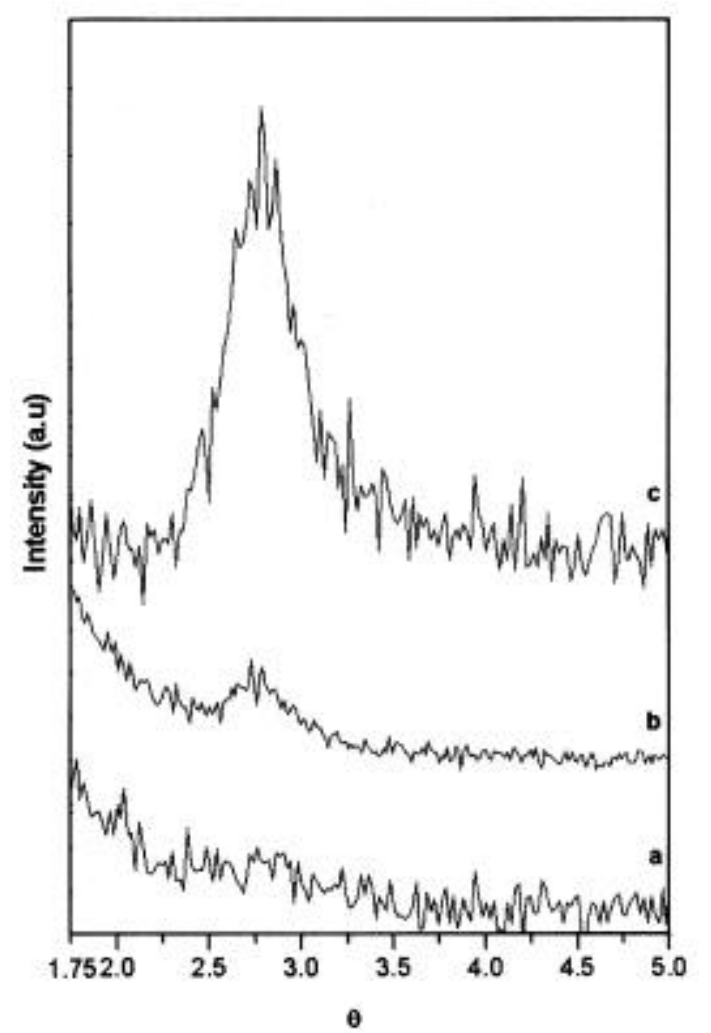

Figure 6. Low-angle X-ray diffraction patterns of SWNTs at various stages of purification: (a) as-synthesised, (b) acid-treated SWNTs and (c) purified SWNTs. 
acid treated and purified treated SWNTs are shown. The $(1,0)$ diffraction line is not observed in the case of as-synthesized SWNTs and appears as a small hump in the case of acid-treated SWNTs. The $(1,0)$ diffraction is observed as a broad intense peak in the case of second hydrogen treated sample. The mean diameter of the purified sample from the $(1,0)$ diffraction line is $1.52 \mathrm{~nm},{ }^{19}$ thus indicating that high quality SWNTs can be obtained by this procedure.

Raman spectroscopy is an established tool to measure the diameter of SWNTs. ${ }^{20,21}$ The G-band and the radial-breathing modes of the SWNTs are strong in intensity while the others Raman modes are weak. ${ }^{21}$ In figure 7 , we show the Raman spectra of the SWNT

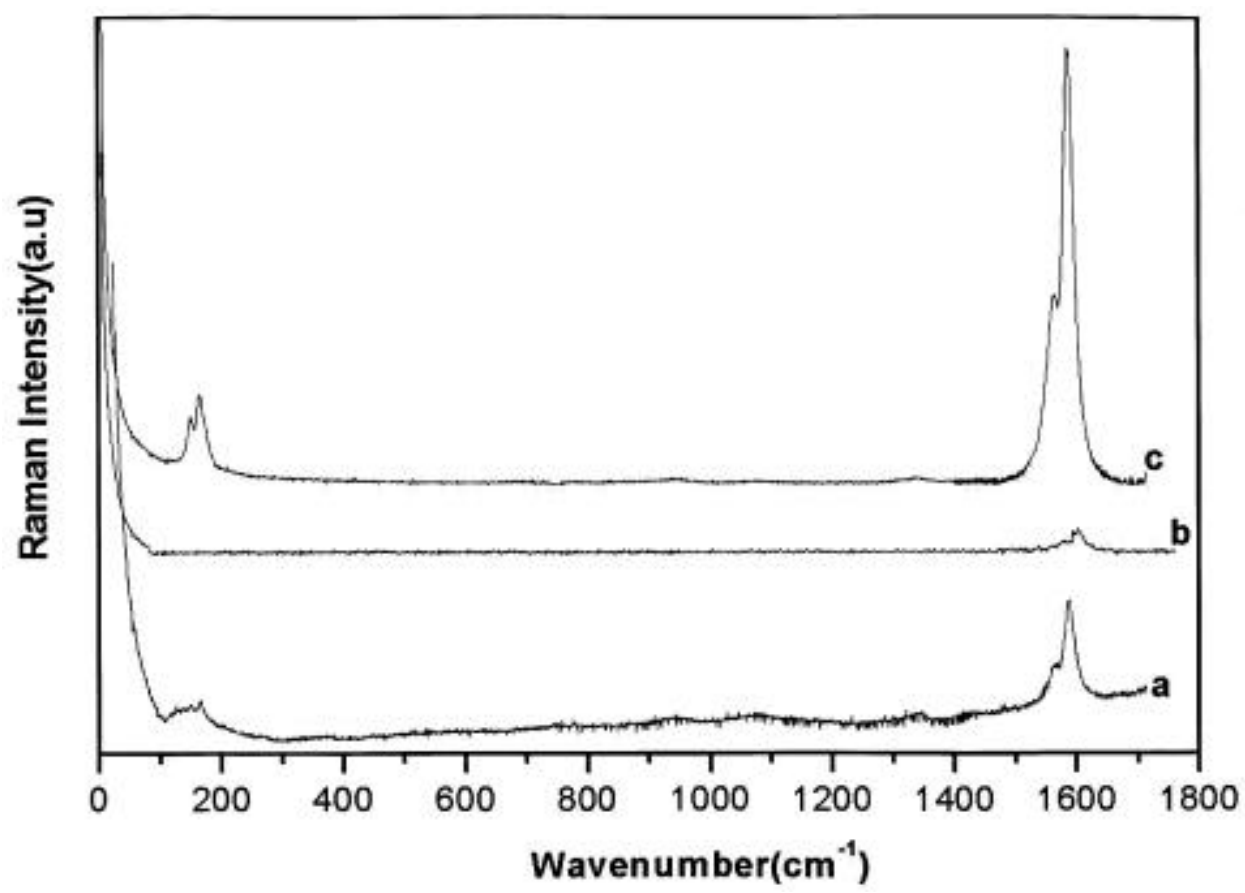

Figure 7. Raman spectra of SWNTs at various stages of purification: (a) assynthesised (sample A), (b) acid-treated SWNTs (sample C) and (c) purified SWNTs (sample D).

sample at various stages of purification. The purity of the SWNTs can be obtained from the intensities from the various bands and from the shape of the D-band. The assynthesized samples (curve a) show a G-band which is split into two with peaks at 1562 and $1586 \mathrm{~cm}^{-1}$. The D-band appears as a broad peak centred at $1343 \mathrm{~cm}^{-1}$. This indicates that the sample contains amorphous carbon. The second order Raman bands appear as weak peaks centred at 941 and $1075 \mathrm{~cm}^{-1}$. The acid treated SWNTs exhibit only a weak $\mathrm{G}$ band $\left(1586 \mathrm{~cm}^{-1}\right)$, which is not split. This can be due to the amorphous carbon covering on the SWNTs, as observed in the SEM images (see figure 3a). The purified sample shows an intense split G-band $\left(1563\right.$ and $\left.1585 \mathrm{~cm}^{-1}\right)$ and a strong radial-breathing mode. The D-band is considerably weaker in intensity and has a sharp shape, which indicates that the sample is pure and does not contain any amorphous carbon. We have 
calculated the diameters of the SWNTs from the radial breathing modes. The diameters calculated from the well-known relation ${ }^{21,22}$ lie in the range $1.32-1.89 \mathrm{~nm}$. This is in good agreement with the calculated diameter distribution obtained from TEM images and lowangle $\mathrm{X}$-ray diffraction.

To get more insight into the effect of high temperature hydrogen treatment, we subjected fuctionalized SWNTs to hydrogen treatment, especially to find out whether the hydrogen etches away the functional groups present on the surface. We observed that hydrogen treatment of chlorinated and amine derivatized SWNTs leads to the degradation of the SWNTs. This indicates that the functional groups in highly fuctionalized SWNTs cannot be removed by high temperature hydrogen treatment.

\section{Conclusion}

The present study demonstrates that high temperature hydrogen treatments can be used to purify SWNTs produced by the arc discharge method. Extensive high temperature hydrogen treatment removes the amorphous carbon and cleans the surface of the SWNTs. The SWNTs purified by this method are far superior in quality compared to those purified by other methods, as they do not contain any hollow onion-like structures. The method may be of value in large-scale purification of SWNTs.

\section{Acknowledgements}

The authors thank Dr Chandrabhas Narayana and Ms G Kavitha in helping with the Raman measurements. The authors also thank Prof. CNR Rao for his suggestions, guidance and encouragement. The authors thank Defence Research and Development Organization for support.

\section{References}

1. Rao C N R, Satishkumar B C, Govindaraj A and Nath M 2001 Chem. Phys. Chem. 278

2. Haddon R C (ed.) 2002 Acc. Chem. Res. 35997 (special issue on carbon nanotubes)

3. Saito R, Dresselhaus G and Dresselhaus M S 1998 Physical properties of carbon nanotubes (London: Imperial College Press)

4. Iijima S and Ichihashi T 1993 Nature (London) 363603

5. Bethune D S, Kiang C H, de Vries M S, Gorman G, Savoy R, Vazques J and Beyers R 1993 Nature (London) $\mathbf{3 6 3} 605$

6. Thess A, Lee R, Nikolaev P, Dai H, Petit P, Robert J, Xu C, Lee Y H, Kim S G, Rinzler AG, Colbert D T, Scuseria G E, Tománek, Fischer J E and Smalley R E 1996 Science 273483

7. Journet C, Maser W K, Bernier P, Loiseau A, Lamy de la Chapelle M, Lefrant S, Deniard P, Lee R and Fischer J E 1997 Nature (London) 388756

8. Govindaraj A and Rao C N R 2002 Pure Appl. Chem. 741571

9. Tohji K, Goto T, Takahashi H, Shinoda Y, Shimizu N, Jeyadevan B, Matsuoka I, Saito Y, Kasuya A, Ohsuna T, Hiraga K and Nishina Y 1996 Nature (London) 383679

10. Tohji K, Goto T, Takahashi H, Shinoda Y, Shimizu N, Jeyadevan B, Matsuoka I, Saito Y, Kasuya A, Ito S and Nishina Y 1997 J. Phys. Chem. B101 1974

11. Bandow S, Rao A M, Williams K A, Thess A, Smalley R E and Eklund P C 1997 J. Phys. Chem. B101 8839

12. Rinzler A G, Liu J, Nikolaev P, Huffman C B, Rodrígues-Macías, Boul P J,Lu A H, Heymann D, Colbert D T, Lee R S, Fischer J E, Rao A M, Eklund P C and Smalley R E1998Appl. Phys. A67 29

13. Shelimov K B, Esenaliev R O, Rinzler A G, Huffman C B and Smalley R E1998 Chem. Phys. Lett. 282429 
14. Dujardin E, Ebbesen T W, Krishnan A and Treacy M M J 1998 Adv. Mater. 10611

15. Dillon A C, Gennett T, Jones K M, Alleman J L, Parilla P A and Heben M J 1999Adv. Mater. 111354

16. Zimmerman J L, Bradley R K, Huffman C B, Hauge R H and Margrave J L 2000 Chem. Mater. 121361

17. Chiang I W, Brinson B E, Smalley R E, Margrave J L and Hauge R H 2001 J. Phys. Chem. B105 1157

18. Martínez M T, Callejas M A, Benito A M, Maser W K, Cochet M, Andrés J M, Schreiber J, Chauvet O and Fierro J L G 2002 Chem. Commun. 20021000

19. The diameter of the SWNTs can be calculated by using the formula $\langle D\rangle=\left(d_{11} / \cos \theta\right)-$ $0 \cdot 312 \mathrm{~nm}$

20. Rao A M, Richter E, Bandow S, Chase B, Eklund P C, Williams K A, Fang S, Subbaswamy K R, Menon M, Thess A, Smalley R E, Dresselhaus G and Dresselhaus M S 1997 Science 275 187

21. Dresselhaus M S and Eklund P C 2000 Adv. Phys. 49705

22. The diameters of the SWNTs were calculated using the relationship: $D=248 \mathrm{~cm}^{-1} / \omega\left(\mathrm{in} \mathrm{cm}^{-1}\right)$ 